\title{
Boson-like quantum dynamics of association in ultracold Fermi gases
}

\author{
I. Tikhonenkov and A. Vardi \\ Department of Chemistry, Ben-Gurion University of the Negev, P.O.B. 653, Beer-Sheva 84105, Israel
}

\begin{abstract}
We study the collective association dynamics of a cold Fermi gas of $2 N$ atoms in $M$ atomic modes into a single molecular bosonic mode. The many-body fermionic problem for $2^{M}$ amplitudes is effectively reduced to a dynamical system of $\min \{N, M\}+1$ amplitudes, making the solution no more complex than the solution of a two-mode Bose-Einstein condensate and allowing realistic calculations with up to $10^{4}$ particles. The many-body dynamics is shown to be formally similar to the dynamics of the bosonic system under the mapping of boson particles to fermion holes, producing collective enhancement effects due to many-particle constructive interference. Dissociation rates are shown to enhance as the number of particles whereas association rates are enhanced as the number of holes, leading to boson-like collective behavior.
\end{abstract}

The role of Bose stimulation in the dynamics of coupled atomic and molecular Bose-Einstein condensates (BECs) has been extensively studied since the first theoretical proposals for the conversion of atomic condensates into molecular BECs [1, 2, 3, 4, 5, 6]. It has been shown that the entire condensate will undergo large amplitude coherent oscillations between atoms and molecules. The Bose-enhanced oscillation frequency is predicted in meanfield theory to scale as $\sqrt{N} g$ where $g$ is the single-particle atom-molecule coupling frequency and $N$ is the total number of condensate particles. Quantum-field effects slightly modify the effective frequency to $(\sqrt{N} / \ln N) g$ and introduce collapse and revival of the coherent oscillations due to interparticle entanglement [3, 5]. Yet despite these modifications the collective behaviour remains significantly different than the single pair dynamics, indicating the dramatic effect of quantum statistics on the many-body dynamics.

Experimentally, the effort towards a molecular BEC and the realization of the theoretical predictions, was made using the techniques of Raman photoassociation 7] and Feshbach resonance magnetoassociation [8, 9, 10]. Unexpectedly, the first molecular condensates were produced using the Feshbach method from nearly degenerate gases of fermion atoms 11, 12, 13. which are more stable against vibrational quenching than their bose-atom counterparts. Molecular BECs made of boson constituent atoms came a close second [14, 15].

The possibility of coupling a nearly degenerate atomic Fermi gas with a molecular BEC raises interesting questions about the nature of the ensuing collective dynamics. At first site it appears that the dynamics of associating fermions should be drastically different than the association dynamics of bosons, since the former are subject to Pauli blocking as compared to the Bose stimulation affecting the time evolution of bosons. However, it has been previously noted that collective effects in fermion systems can mimik bosonic stimulation in four-wave mixing [16, 17], where the boson-like behaviour is attained from various pathways adding up constructively [16]. Moreover, few-particle numerical results for the association of a Fermi-Bose mixture of atoms [18], indicate some similarity with the purely bosonic case, in that collective Rabi-like oscillations and Rapid adiabatic passage are observed in both cases. Better understanding of this boson-like behaviour, allowing for the simplification of calculations and illuminating its origin is highly desirable.

In this work we study the association dynamics of an atomic fermion gas into a degenerate molecular bose gas. We show that the many-fermion problem which is apparently much more obstruse than the corresponding twomode bosonic problem (or than the Fermi-Bose mixture case), can be effectively reduced to an $(N+1) \times(N+1)$ system of dynamical equations, where $N$ is the total number of pairs (i.e. the sum of the molecule number and half the atom number). The complexity of the system is thus identical to that of the dynamical equations obtained for two-mode bose association in its number-state representation 3, 51, allowing for numerical calculations with up to $N=10^{4}$ particles with current computation power, as opposed to characteristically $N=10$ particles in Ref. [18].

Furthermore, we show formally that in the limit of large $N$ and $M$ where the number of fermion modes $M$ is equal to the number of particles $N$, the structure of the fermionic system is a 'mirror image' of the bosonic system, producing precisely the same dynamics for fermion dissociation as was attained for boson association. Similarly the dynamics of association into fermions reproduces the known results for boson dissociation, including its modulational instability [5]. It is evident from our model that the enhancement effects come from adding up the various pathways connecting states with $N-n$ molecules and $n$ dissociated pairs with states containing one more (or one less) molecule and one less (or one more) dissociated pair. Thus, the origin of the nonlinear collective behaviour is found to be identical to the four-wave mixing case [16].

We consider the association of a Fermi gas of atoms into Bose molecules. The interaction representation 

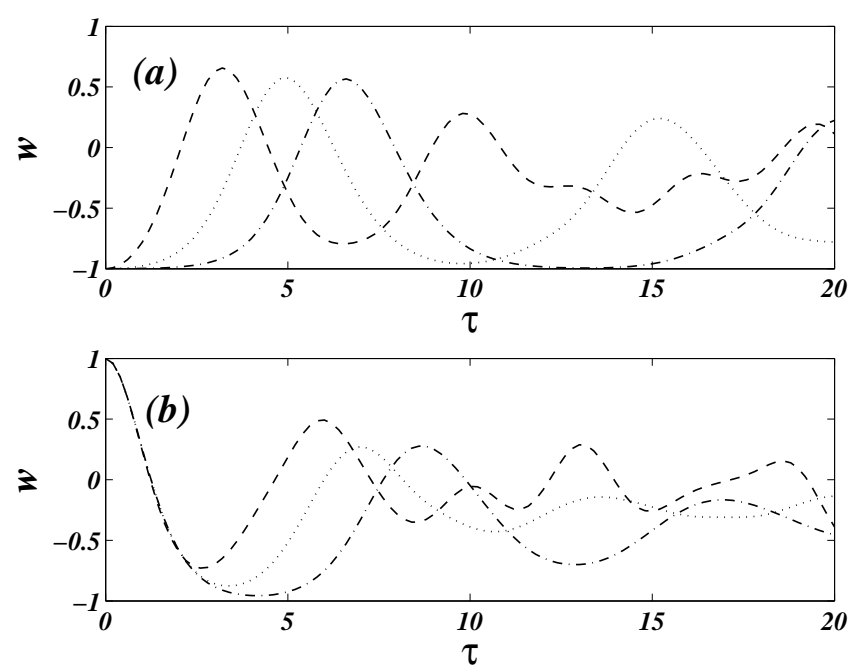

FIG. 1: Collective dynamics of association of an atomic Fermion gas into a molecular BEC (a) and dissociation of a molecular BEC into a degenerate Fermi gas of atoms (b). Particle pair numbers are fixed to $N=5$ (dashed line), $N=50$ (dotted line), and $N=500$ (dash-dotted line) pairs. The number of fermion modes is set equal to the number of particles.

Hamiltonian reads

$$
\begin{aligned}
H= & \sum_{\mathbf{k}, \sigma} \epsilon_{\mathbf{k}} c_{\mathbf{k}, \sigma}^{\dagger} c_{\mathbf{k}, \sigma}+\sum_{\mathbf{k}} \mathcal{E}_{\mathbf{k}} b_{\mathbf{k}}^{\dagger} b_{\mathbf{k}} \\
& +\left(g \sum_{\mathbf{k}} c_{\mathbf{k}, \uparrow} c_{\mathbf{k}^{\prime}, \downarrow} b_{\mathbf{k}+\mathbf{k}^{\prime}}^{\dagger}+\text { H.c. }\right),
\end{aligned}
$$

where $\epsilon_{\mathbf{k}}=\hbar^{2} k^{2} / 2 m$ is the kinetic enery for an atom with mass $m, \mathcal{E}_{\mathbf{k}}$ is the molecular energy containing both kinetic and binding conributions, $c_{\mathbf{k}, \sigma}$ are the annihilation operators for the atoms, obeying the usual Fermi anticommutation relation, $b_{\mathbf{k}}$ are the molecular annihilation operators, commuting with $c_{\mathbf{k}, \sigma}$ and obeying bosonic commutation relations, and $g$ is the strength of the atommolecule coupling. Since the pairing instability occurs predominantly for colliding atom pairs whose center of mass momentum is zero [19], as confirmed by the formation of a molecular BEC in the experiments of Refs. $[11,12,13$, we use the single molecular mode approximation 20, 21], assuming that only singlet pairs of opposite momenta are associated, resulting in molecules in the lowest energy molecular mode. Moreover, since the Fermi energy is characteristically very small compared with the atom-molecule coupling frequency, we can safely ignore the variation in $\epsilon_{\mathbf{k}}$. This approximation is tantamount to assuming that the motional timescale of the atoms is much longer than the timescale of atom-molecule conversion [18] and is verified by the nearly complete conversion of Fermi atoms to Bose molecules in Feshbach sweep experiments [11, 12, 13] (we note paranthetically that the variation in $\epsilon_{\mathbf{k}}$ can be easily incorporated into our model by proper renormalization of the amplitudes). The resulting rotating wave Hamiltonian is

$H=\Delta\left(\frac{1}{2} \sum_{\mathbf{k}, \sigma} c_{\mathbf{k}, \sigma}^{\dagger} c_{\mathbf{k}, \sigma}-b_{0}^{\dagger} b_{0}\right)+\left[g \sum_{\mathbf{k}} c_{\mathbf{k}, \uparrow} c_{-\mathbf{k}, \downarrow} b_{0}^{\dagger}+H . c.\right]$

where $\Delta=2 \epsilon_{k_{F}}-\mathcal{E}_{0}$. In what follows we shall assume that $\Delta=0$, i.e. we consider the case of resonant coupling between atoms and molecules, retaining only the interaction Hamiltonian. Since the Hamiltonian (2) conserves the number of atoms, i.e. $2 n+\sum_{\sigma, \mathbf{k}} n_{\sigma, \mathbf{k}}=2 N$ where $n$ is the numeber of molecules, $n_{\mathbf{k}, \sigma}$ is the number of atoms in state $\mathbf{k}, \sigma$ and $N$ is the (conserved) total number of pairs, we can fix $N$ and expand the quantum state of the system in pair number states:

$$
|\psi\rangle=\sum_{n=0}^{\min \{N, M\}} \sum_{\left\{n_{k}\right\}} C_{N-n, n_{1}, \ldots, n_{M}}(t)\left|N-n, n_{1}, \ldots, n_{M}\right\rangle
$$

where $k$ denotes some labeling of the $M$ available momentum states, $n_{k}=0,1$ are fermionic occupation numbers for the free atom states (where $n_{k}=1$ denotes a singlet pair of particles occupying the momentum states $\mathbf{k},-\mathbf{k}$ ) and the summation over $\left\{n_{k}\right\}$ for any given $n$ is over all nonordered combinations of $n$ atom pairs among $M$ states, so that $\sum_{k=1}^{M} n_{k}=n$. The effective phase-space for the problem can thus be classified into $\min \{N, M\}+1$ manifolds characterized by the number of dissociated pairs $n=0,1, \ldots, \min \{N, M\}$. For each $n$, the size of the corresponding manifold is the number of orderings of $n$ pairs in $M$ states given by the binomial coefficient $\left(\begin{array}{l}M \\ n\end{array}\right)$. It is immediately seen that the interaction Hamiltonian couples each state in the manifold with $n$ dissociated pairs to $M-n$ states having $n+1$ dissociated pairs and to $n$ states with $n-1$ free atom pairs. Substituting the expansion (3) into the Schrödinger equation $i \hbar \partial_{t}|\psi\rangle=H|\psi\rangle$, with the Hamiltonian (11), we obtain:

$$
\begin{aligned}
i \dot{C}_{N-n, n_{1}, \ldots, n_{M}}= & g(\sqrt{N-(n-1)} \\
& \times \sum_{k=1}^{M} \delta_{1, n_{k}} C_{N-(n-1), n_{1}, \ldots, n_{k}-1, \ldots, n_{M}} \\
& +\sqrt{N-n} \\
& \left.\times \sum_{k=1}^{M} \delta_{0, n_{k}} C_{N-(n+1), n_{1}, \ldots, n_{k}+1, \ldots, n_{M}}\right)
\end{aligned}
$$

where $n=0, \ldots, \min \{N, M\}$. These equations of motion can be put into the matrix form:

$i \dot{\mathbf{C}}_{n}=g\left(\sqrt{N-n+1} D_{n}^{n-1} \mathbf{C}_{n-1}+\sqrt{N-n} D_{n}^{n+1} \mathbf{C}_{n+1}\right)$

where $\mathbf{C}_{n}(t)$ is a column vector of all the $\left(\begin{array}{l}M \\ n\end{array}\right)$ amplitudes corresponding to the possible arrangements of the $n$ atom 
pairs among the $M$ available fermion modes. The matrices $D_{I}^{J}$ are $\left(\begin{array}{c}M \\ I\end{array}\right) \times\left(\begin{array}{c}M \\ J\end{array}\right)$ dimensional and contain only unit and zero elements. Their explicit form is determined by the ordering of elements in the vectors $\mathbf{C}_{I}$ and $\mathbf{C}_{J}$.

The system (5) contains equations for $2^{M}$ amplitudes, making effective dynamical calculations restricted to limited numbers of particle (characteristically no more than 20 particles with current computation power). However, it is clear that the high symmetry of the system should produce conservation laws that would allow its simplification. In fact, since all the states corresponding to various atom ordering within each $\left(\begin{array}{c}M \\ n\end{array}\right)$ manifold of $N-n$ molecules are equivalent (their coupling strength to states with one more or one less molecule are equal and each is coupled to the same number of states above and below it), there are $\left(\begin{array}{l}M \\ n\end{array}\right)-1$ constraints in each such manifold, leaving effectively only $\min \{N, M\}+1$ free amplitudes. Consequently, an enormous reduction in the dimensionality of the system is attained by multiplication of Eq. (5) by a row vector $\mathbf{u}_{n, M}$ of $\left(\begin{array}{l}M \\ n\end{array}\right)$ unit elements (thus summing over all amplitudes in the manifold of $n$ dissociated pairs) and use of the identities:

$$
\begin{gathered}
\mathbf{u}_{n, M} D_{n}^{n+1}=(n+1) \mathbf{u}_{n+1, M}, \\
\mathbf{u}_{n, M} D_{n}^{n-1}=(M-n+1) \mathbf{u}_{n-1, M} .
\end{gathered}
$$

The resulting equations of motion read

$$
\begin{aligned}
i \dot{\alpha}_{n}= & g\left(\sqrt{N-n+1} \sqrt{n(M-n+1)} \alpha_{n-1}\right. \\
+ & \left.\sqrt{N-n} \sqrt{(M-n)(n+1)} \alpha_{n+1}\right)
\end{aligned}
$$

where

$$
\alpha_{n} \equiv \frac{\mathbf{u}_{n, M} \mathbf{C}_{n}}{\sqrt{\left(\begin{array}{c}
M \\
n
\end{array}\right)}}=\frac{\sum_{\left\{n_{k}\right\}} C_{N-n, n_{1} \ldots, n_{M}}}{\sqrt{\left(\begin{array}{c}
M \\
n
\end{array}\right)}}, \sum_{k=1}^{M} n_{k}=n,
$$

is the sum over all $\left(\begin{array}{c}M \\ n\end{array}\right)$ amplitudes with $N-n$ molecules and $n$ free atom pairs, corresponding to the various orderings of the atoms, normalized by the number of permutations of $n$ atoms in $M$ states. The average number of molecules, provided that all amplitudes in the $n$-th manifold are equivalent (i.e. under the restriction that initial conditions do not break this equivalence), is calculated according to

$$
\begin{aligned}
\left\langle b_{0}^{\dagger} b_{0}\right\rangle= & \sum_{m=0}^{\min \{N, M\}}(N-n) \sum_{\left\{n_{k}\right\}}\left|C_{N-n, n_{1} \ldots, n_{M}}\right|^{2} \\
& =\sum_{m=0}^{\min \{N, M\}}(N-n)\left|\alpha_{n}\right|^{2}
\end{aligned}
$$

The size of the system [8] is thus reduced to $\min \{N, M\}+1$, exactly as would be obtained for the
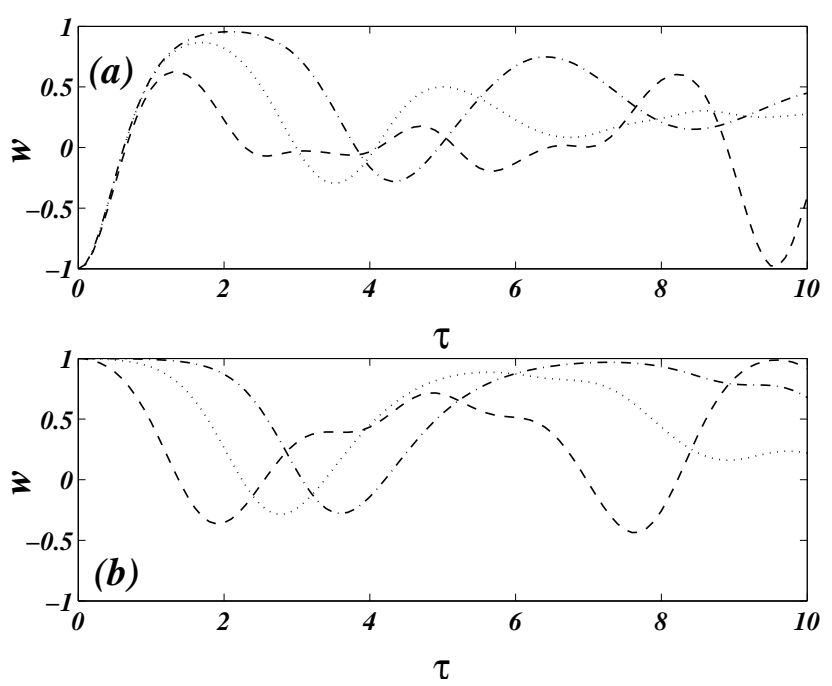

FIG. 2: Collective dynamics of association (a) and dissociation (b) in a two-mode atom-molecule BEC. Particle numbers are fixed to $N=5$ (dashed line), $N=50$ (dotted line), and $N=500$ (dash-dotted line) pairs. The number of fermion modes is set equal to the number of particles.

equivalent two mode bosonic system, allowing numerical results with up to $10^{4}$ particles. The physical interpretation of Eqs. (8) is quite striking. It shows that in addition to the usual bosonic factors of $\sqrt{N-n+1}$ for association and $\sqrt{N-n}$ for dissociation, originating from the molecular bosonic field operator $b_{0}$, the association rate depends on the number of pairs in the initial state (a trivial factor originating from having $n$ pairs which can associate) but is also enhanced by the number of holes in the target state. Similarly, the rate of dissociation (i.e. the annihilation of a hole) scales with the number of holes in the initial state but also with the number of particles (or more precisely, particle-pairs) in the target state.

The resulting dynamics should thus be surprisingly similar to the dynamics of an atom-molecule condensate. It is illuminating to compare Eq. (8) to the $(N+1) \times$ $(N+1)$ number-state representation of the Schrödinger equation for a two-mode atom-molecule BEC [3, 5]

$$
\begin{aligned}
i \dot{\beta}_{n}= & g\left(\sqrt{N-n+1} \sqrt{2 n(2 n-1)} \beta_{n-1}\right. \\
& \left.+\sqrt{N-n} \sqrt{(2 n+2)(2 n+1)} \beta_{n+1}\right),
\end{aligned}
$$

where $n=0, \ldots, N$ and the average molecule number is $\left\langle b^{\dagger} b\right\rangle=\sum_{n=0}^{N}(N-n)\left|\beta_{n}\right|^{2}$. Examination of Eq. (11) shows that both the association rate and the dissociation rate are enhanced as the number of atoms in the target state. Moreover, it is evident that up to factors of two, the main difference between the fermion equation (8) and the boson equation (11) is in the enhancement factors where particle number terms $n$ in the boson case are replaced by hole number terms $M-n$ for fermions. It is therefore clearly evident that when $N=M$ (i.e. the 
number of pair states is equal to the number of pairs) the dynamics of fermion association (dissociation) should be qualitatively very similar to the dynamics of boson dissociation (association), up to a factor of two in the pertinent rates.

This observation is confirmed by numerical integration of Eq. (8) and Eq.(11) for fermions and bosons respectively. In Fig. 1 and Fig. 2 we plot the expectation value of the pair-number difference $w=2\left\langle b_{0}^{\dagger} b_{0}\right\rangle / N-1$ as a function of the rescaled time $\tau=\sqrt{2 N} g$ t. Initial conditions had all free fermion atoms in (a) or all molecules in (b). The fermion results in Fig. 1 mirror the boson results of Fig. 2 (identical to Refs. 3, [5]) in that the dynamics of fermion association closely resembles the dynamics of boson dissociation and vice versa. The similarities include the $\sqrt{N} / \ln N$ scaling of the atom-molecule oscillation frequency [5] and the dynamical instability of the fermion atoms to molecule formation, noted previously in mean-field studies [20, 21]. This instability turns out to be the mirror image of the familiar modulational instability of the molecular mode in the two-mode BEC dynamics [5]. As expected, a factor of two exists in the characteristic frequencies.

The mirror symmetry between fermion and boson association dynamics in the limit of large $N=M$ is in fact exact. To show this we note that for large $N$ the boson equations of motion (11) for the amplitudes $\beta_{N-m}$ may be written as

$$
\begin{aligned}
i \dot{\beta}_{N-n}= & 2 g\left(\sqrt{n+1}(N-n) \beta_{N-n-1}\right. \\
& \left.+\sqrt{n}(N-n+1) \beta_{N-n+1}\right),
\end{aligned}
$$

where we have neglected some terms of order $1 \ll 2 N$. Mapping $\beta_{N-n} \rightarrow \beta_{n}$ we obtain that

$$
\begin{aligned}
i \dot{\beta}_{n}= & 2 g\left((N-n+1) \sqrt{n} \beta_{n-1}\right. \\
& \left.+(N-n) \sqrt{n+1} \beta_{n+1}\right),
\end{aligned}
$$

which is identical up to a factor of two, to the fermion equation (8) when $N=M$. This factor of two emanates from having a single atomic mode with $2 n$ particles in the bosonic problem, making it the analog of a degenerate optical parametric amplifier (OPA), as opposed to having $n$ particles each of spin $\uparrow$ and spin $\downarrow$ atoms in the fermion problem. The correspondence is thus even better with the nondegenerate OPA-like association of a two species BEC

$$
H=g\left(a_{1} a_{2} b^{\dagger}+b a_{2}^{\dagger} a_{1}^{\dagger}\right),
$$

where $a_{1}, a_{2}$ and $b$ are all boson annihilation operators. The number-state representation of the Schrödinger equation with the Hamiltonian (14) maps exactly to Eq. (13) under $\beta_{N-n} \rightarrow \beta_{n}$, without the multiplying factor of two and the restriction of large $N$. The resulting dynamics of this boson problem at any $N$ is thus just the mirror image of Fig. 1.
The dynamics of the fermion amplitude corresponding to $n$ atom pairs (and $M-n$ hole pairs) thus maps precisely for $N=M$, to the exact quantum dynamics of the two-species boson amplitude corresponding to $N-n$ atom pairs. At the limit of large $N$, the two-species boson dynamics reproduces the single atomic mode boson dynamics with a factor of two in the characteristic timescales. One can thus solve the fully quantum fermion problem by simply solving the nondegenerate (two atomic species) two-mode BEC problem, and mapping boson amplitudes with $n$ particles to fermion amplitudes with $n$ holes, or at the limit of large $N$, solve the degenerate (single atomic specie) two-mode BEC problem, carry out the same particle-hole mapping, and divide the timescale by two.

To conclude, the apparently complex dynamics of an ultracold Fermi gas coupled to a molecular BEC can be greatly simplified due to the inherent symmetry of the problem. We have obtained a system of dynamical equations for $N+1$ effective amplitudes enabling an improvement of three orders of magnitude in the total particle number of a realistic calculation. The structure of this system provides great insight into the origin of bosonlike collective behavior. It demonstrates that dissociation is enhanced as the number of particles whereas association is enhanced as the number of holes, due to constructive interference of various pathways between states with different molecule number. Formal identity between fermion and boson dynamics was shown to exist when $N=M$ and confirmed by numerical calculations. This equivalence can serve as an important tool in further studies of nearly degenerate fermion-boson systems.

We thank Ehoud Pazy for valuable discussions. This work was supported in part by grants from the U.S.-Israel Binational Science Foundation (grant No. 2002214), the Minerva Foundation through a grant for a Minerva Junior Research Group, and the Israel Science Foundation for a Center of Excellence (grant No. 8006/03).

[1] E. Timmermans et al., Phys. Rev. Lett. 83, 2691 (1999).

[2] F. A.van Abeelen and B. J. Verhaar, Phys. Rev. Lett. 83, 1550 (1999).

[3] J. Javanainen and M. Mackie, Phys. Rev. A 59, R3186 (1999).

[4] D. J. Heinzen et al., Phys. Rev. Lett. 84, 5029 (2000).

[5] A. Vardi et al., Phys. Rev. A 64, 063611 (2001).

[6] M. G. Moore and A. Vardi, Phys. Rev. Lett. 88, 160402 (2002).

[7] R. Wynar et al., Science 287, 1016 (2000).

[8] E. A. Donley et al., Nature 412, 295 (2001).

[9] N. R. Clausessen et al., Phys. Rev. Lett. 89, 010401 (2002).

[10] E. A. Donley et al., Nature 417, 529 (2002).

[11] S. Jochim et al., Science 302, 2101 (2003).

[12] M. Greiner et al., Nature 426, 537 (2003). 
[13] M. W. Zwierlein et al., Phys. Rev. Lett. 91, (250401)(2003).

[14] K. Xu et al., Phys. Rev. Lett. 91, 210402 (2003).

[15] T.Mukaiyama et al., Phys. Rev. Lett. 92, 180402 (2004).

[16] M. G. Moore and P. Meystre, Phys. Rev. Lett. 86, 4199 (2001).

[17] W. Ketterle and S. Inouye, Phys. Rev. Lett. 86, 4203 (2001).
[18] O. Dannenberg et al., Phys. Rev. Lett. 91, 210404 (2003).

[19] G. D. Mahan, Many-Particle Physics (Plenum, New York, 2000).

[20] J. Javanainen et al., Phys. Rev. Lett. 92, 200402 (2004).

[21] R. A. Barankov and L. S. Levitov, cond-mat/0405178 (2004). 